\title{
3D CAD model reconstruction of a human femur from MRI images
}

\author{
Mohammed RADOUANI \\ ENSAM, National Higher School of Engineering \\ Moulay Ismail University Meknès, Morocco \\ Benaissa EL FAHIME \\ ENSAM, National Higher School of Engineering \\ Moulay Ismail University \\ Meknès, Morocco
}

\begin{abstract}
Medical practice and life sciences take full advantage of progress in engineering disciplines, in particular the computer assisted placement technique in hip surgery. This paper describes the three dimensional model reconstruction of human femur from MRI images. The developed program enables to obtain digital shape of $3 D$ femur recognized by all CAD software and allows an accurate placement of the femoral component. This technic provides precise measurement of implant alignment during hip resurfacing or total hip arthroplasty, thereby reducing the risk of component malpositioning and femoral neck notching.
\end{abstract} femur

Keywords-biomechanic; MRI imaging; $3 D$ reconstructionl;

\section{INTRODUCTION}

Collaborations between the medical and engineering communities in design and implantation of total joint replacements provide a means to restore function to a degraded human joint.

In case of hip surgery, Computer Assisted Surgery system needs the use of 3D CAD model of femur as reference shape to plan the virtual surgery. This model can be reconstructed using the images generated from scanners and enables observing the layers of the tissue shown in the images on a pixel by pixel level and in the context of the 3D structure of the human body.

Magnetic resonance imaging is a valuable imaging technique used primarily in medical settings to produce high quality images of the inside of the human body [1].

The clear anatomical images which can be produced in any plane, coupled with inherent safety for the patient and longterm cost effectiveness have promoted the use and availability of MRI around the world.

Automatic treatment techniques for visualization, interpretation and exploitation of these complex images have an enormous beneficial impact on clinical practice and research, by decreasing dramatically the manual effort which must otherwise be devoted [2]. In this work we develop a processing approach of MRI images for automatic reconstruction of a $3 \mathrm{D}$ model of the human femur.

\author{
Youssef AOURA \\ ENSAM, National Higher School of Engineering \\ Moulay Ismail University \\ Meknès, Morocco \\ Latifa OUZIZI \\ ENSAM, National Higher School of Engineering \\ Moulay Ismail University \\ Meknès, Morocco
}

\section{MRI IMAGES TREATMENT OF THE FEMUR}

\section{A. Magnetic Resonance Imaging}

Magnetic resonance imaging is based on the principles of nuclear magnetic resonance (NMR), a spectroscopic technic used by scientists to obtain microscopic chemical and physical information about molecules.

The technique was called magnetic resonance imaging rather than nuclear magnetic resonance imaging (NMRI) because of the negative connotations associated with the word nuclear in the late 1970's.

MRI started out as a tomographic imaging technique, that is it produced an image of the NMR signal in a thin slice through the human body. Each slice had a thickness. This form of imaging is in some respects equivalent to cutting off the anatomy above the slice and below the slice. The slice is said to be composed of several volume elements or voxels. The volume of a voxel is approximately $2 \mathrm{~mm}^{3}$.

The magnetic resonance image is composed of several picture elements called pixels. The intensity of a pixel is proportional to the NMR signal intensity of the contents of the corresponding volume element or voxel of the object being imaged.

\section{B. MRI images treatment}

The process of 3D model reconstruction of femur is described by the following organizational chart:

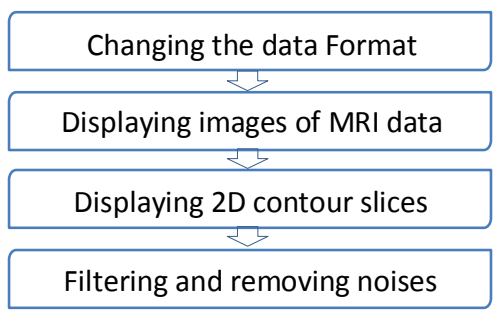

In first step, we have imported the MRI images into MATLAB workspace and saved it in a ".mat" file [3]. Then images are converted from grayscale to indexed ones. This 
procedure makes it possible to treat them as $4 \mathrm{D}$ matrixes. The set of 16 images are used in transversal plan (figure 1).

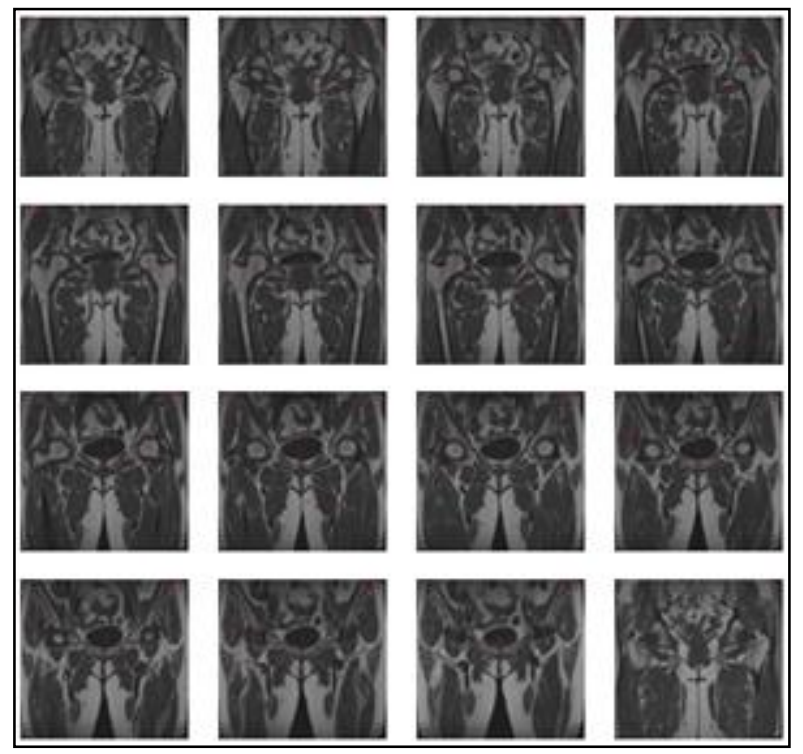

Fig.1. MRI images of femur in sagittal plane.

The whole set of 16 converted images is concatenated one on top of the other into a matrix using cat() function in Matlab software [4].

With this matrix an extra dimension derived from obsolete color maps is added. It can be eliminated using squeeze() function.

The Displaying 2D contour slices step consists on drawing contours in volume slice planes of all images. To eliminate artifacts, we designed the function to select the ROI (Region of Interest), and to convert the pixels out of ROI into the ones with the minimal value (figure 2).



Fig.2. Contours detection of a single layer.

A function to filter the images obtained previously was programmed to eliminate residual noise. An example of the results of this operation is presented in figure 3 .
The parameters which define the way of filtering are determined by the contrast and the color range of the acquired images.

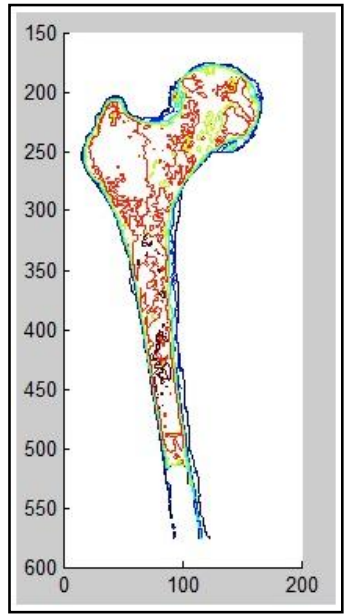

Fig.3. Bone shape extraction using the filtration function.

The code of the designed functions is as follows:

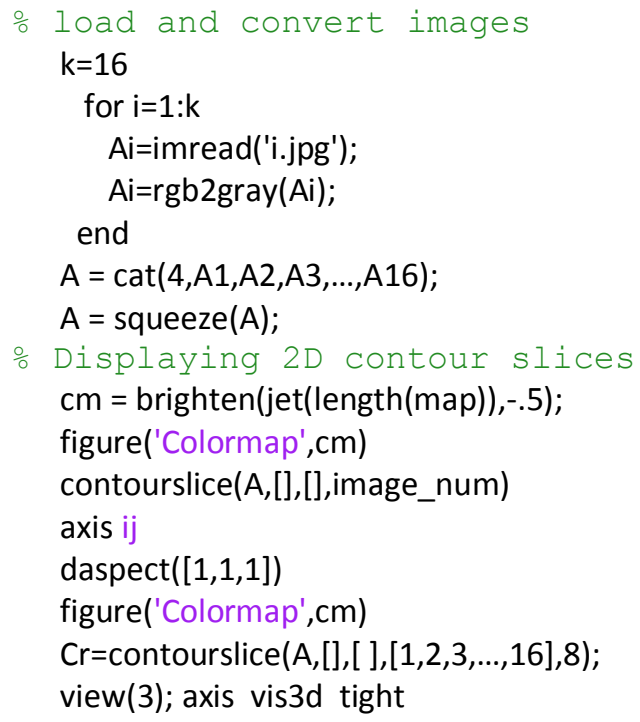

\section{3D RECONSTRUCTION OF FEMUR}

For The 3D automatic reconstruction of the femur a graphical user interface is developed in Matlab GUI environment [4]. This interface is presented in figure 4 and contains four buttons which initiate separate functions of the program:

The first one allows uploading and concatenating images into 3D variables, the whole set of images will be displayed as a MRI film.

Then the images will be filtered by clicking on the second button.

The third button will initiate the $3 \mathrm{D}$ reconstruction by creating and displaying contour slices with the same orientation and sizes as images (figure 5). 


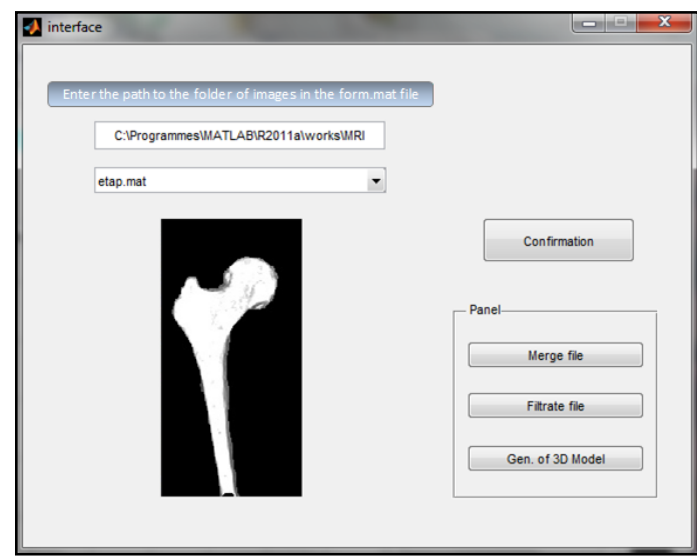

Fig.4. Bone shape extraction using the filtration function.

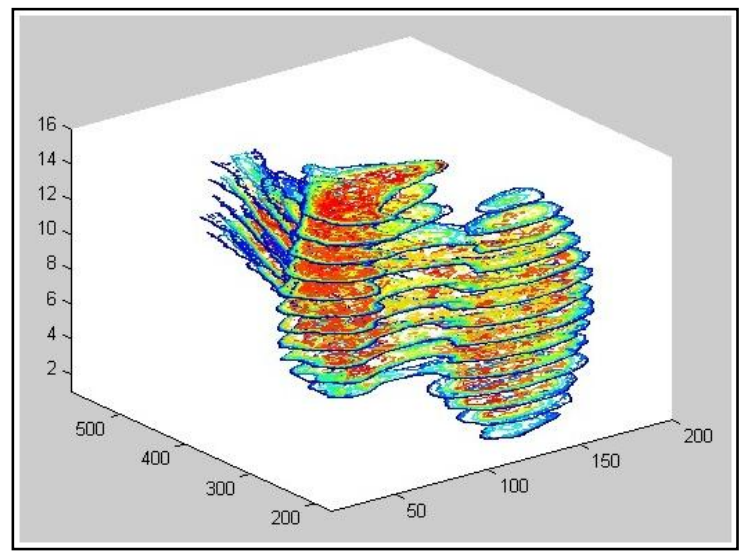

Fig.5. 3D display of contour slices.

At the end, the user has the option to enter the pixel by pixel coordinates of the planes which should divide the structure along the axes into a desired volume for observation.

Matlab editor for displaying 3D images has the ability of rotation and viewing the reconstruction from different angles.

The illumination of the display is set in such a way to show the best contrast in the most practical angles for viewing images (figure 6).

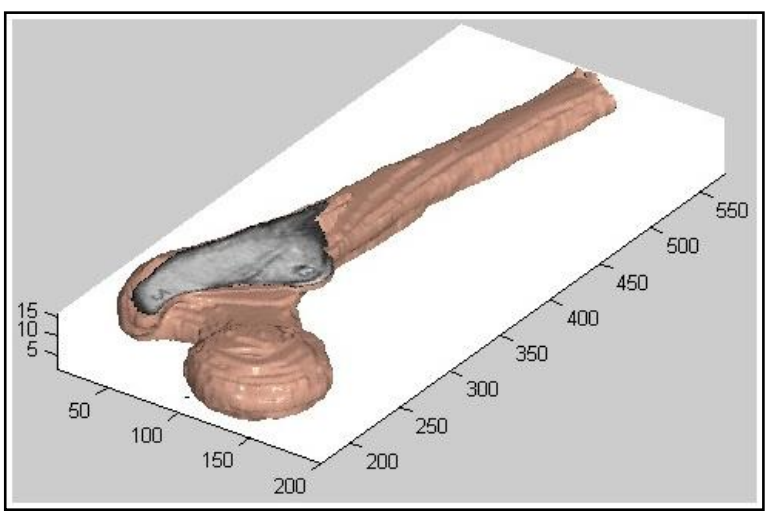

Fig.6. 3D model of the femur.
After having the 3D model of the bone, a STL file format is generated that can be used by all CAD software (figure 7).

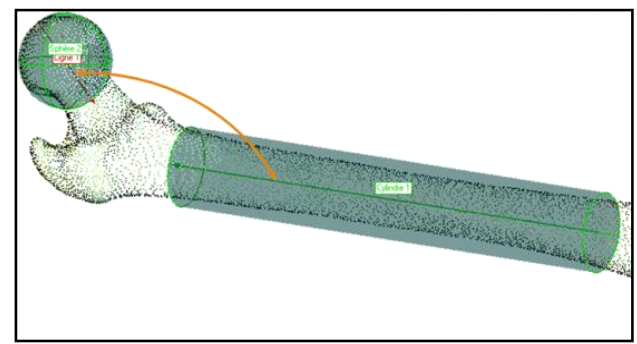

Fig.7. STL foramt of the femur

\section{CONCLUSION}

The computer-assisted placement technique is an accurate and reproducible technique for hip surgery. Implants position may be better achieved for the navigated hips than by the traditional method.

In this study we proposed a method to generate a 3D CAD model of femur from MRI images. The developed technique allows the surgeon to make quickly the necessary decisions regarding the selection and positioning of the implants in 3D and reduce the risk of a misaligned component.

\section{REFERENCES}

[1] W. Birkfellner, "Applied medical image processing : a basic course". Boca Ratton, FL.: CRC Press, 2011.

[2] E. Ukwatta, J. Yuan, M. Rajchl, and A. Fenster, "Efficient global optimization based 3D carotid AB-LIB MRI segmentation by simultaneously evolving coupled surfaces", Med Image Comput Comput Assist Interv, vol. 15, pp. 377-84, 2012.

[3] D. Zhou, W. K. Thompson, and G. Siegle, "MATLAB toolbox for functional connectivity", Neuroimage, vol. 47, pp. 1590-607, Oct 1 2009.

[4] Matlab, Help, "sections: Visualizing MRI data: Volume Visualization Techniques (3-D Visualization) "; Image Processing Toolbox.

\section{AUTHORS PROFILE}

Mohammed RADOUANI is an associate professor at the National Higher School of Engineering (Crafts and Technologies, ENSAM Meknès - Moulay Ismail University, Morocco). He obtained his Ph.D. thesis in Mechanical Engineering from Prestigious training college for teachers and researchers in Technics (ENS of Cachan, University of Paris-south XI France, in 2003) and his Habilitation of supervising scientific research Dissertation from Faculty of Sciences-Meknès in 2009. His research work is dealing with specification and inspection of mechanical systems according to the ISO standards. He is also interested to products numerical engineering.

Youssef AOURA is an associate professor at the National Higher School of Engineering (ENSAM Meknès - Moulay Ismail University, Morocco). He obtained his Ph.D. thesis in Manufacturing Processes from the National Higher School of Engineering (ENSAM - France, in 2004). His research work is dealing with optimisation of new products and manufacturing processes.

Benaissa EL FAHIME obtained his Ph.D. thesis in Mechanical Engineering from the - Faculty of Science and Technology of Fez. His research activities concern the representation of mechanical tolerances in the Bond Graph models of mechatronic systems.

Latifa OUZIZI is an associate professor at the National Higher School of Engineering (ENSAM Meknès - Moulay Ismail University, Morocco). He obtained his Ph.D. thesis in Automatic and Productic from Metz University Metz France, in 2005. His research work is dealing with optimisation of new products and manufacturing processes. 